\title{
para reparación de automóviles
}

ADOLF IDE, Arqto. Dr.-Ing.

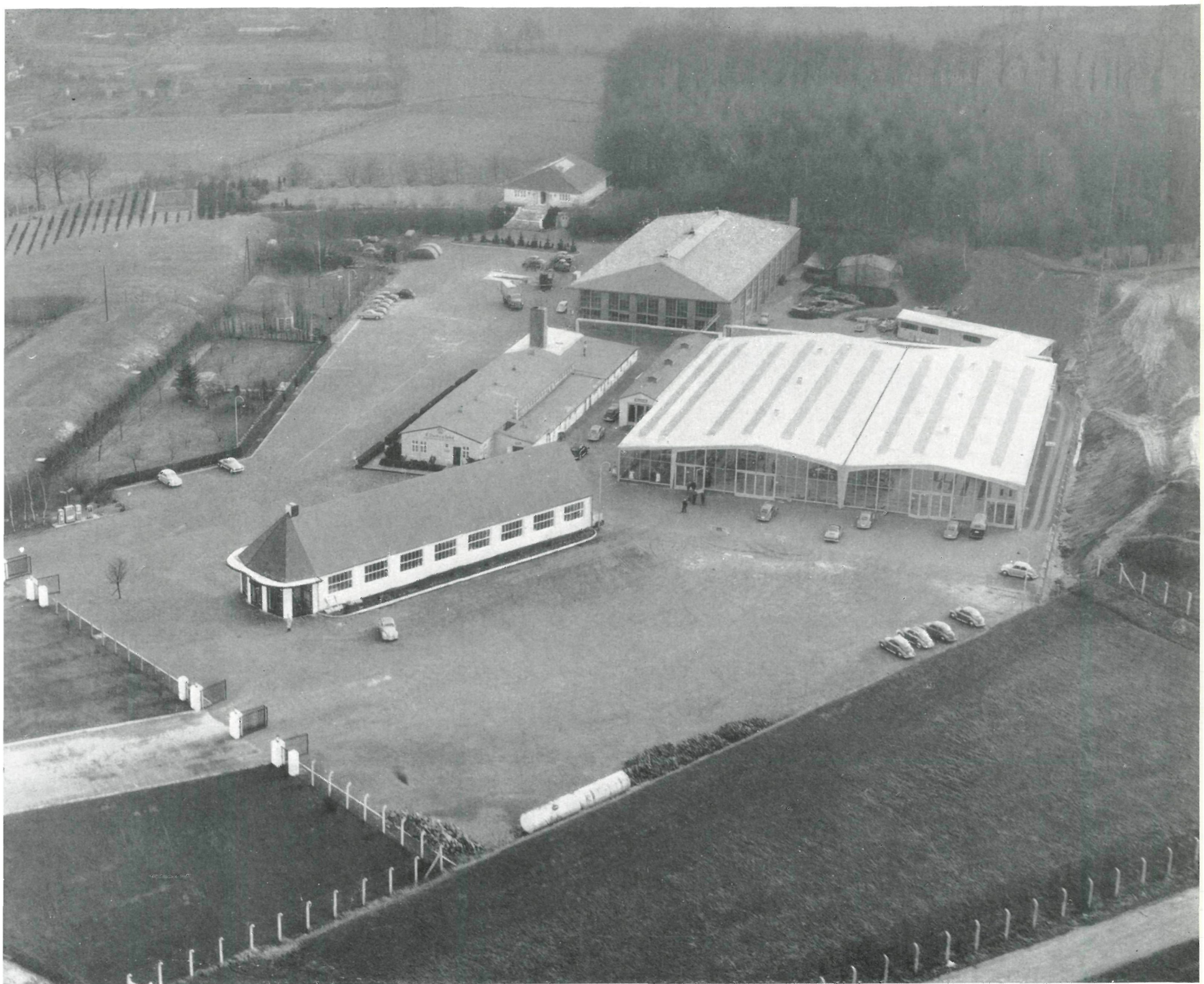

$841 \cdot 18$

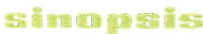

Estos talleres, construidos en Osnabrück (Alemania), están destinados a la empresa Starke \& Sohn, que se dedica a la reparación de automóviles ligeros.

La nave general, de planta rectangular, se ha cubierto con una estructura metálica ligera formada por graciosos pórticos, sobre los que van las correas y el material de cubierta, que es la tradicional chapa ondulada de fibrocemento. Grandes lucernarios para la iluminación cenital corren a lo largo de toda la nave. Los cerramientos laterales y frontales son paños de cristal, lo cual proporciona, como es lógico, una luminosidad extraordinaria.

Todo se ha previsto para que los vehículos que han de ser reparados sigan un circuito continuo desde la entrada hasta la salida del coche ya reparado.

Los soportes de la cubierta son los montantes de los pórticos de dos tramos, que constituyen toda la estructura resistente de la nave. Están perfectamente estudiados todos los problemas de ventilación, iluminación artificial y acondicionamiento 
Los talleres para reparación de automóviles, construidos recientemente en Osnabrück, Alemania, para la casa Starke \& Sohn, fueron proyectados con estructura metálica. Sus cerramientos están constituidos por grandes paños de cristal, de gran espesor, resistentes, que dan paso a la luz natural y, por consiguiente, proporcionan una clara iluminación diurna en su interior.

La gran nave del taller va cubierta con un material llamado Fulgurit. Este material se ha aislado, en su parte interior. La cubierta lleva varios lucernarios continuos que se extienden formando bandas longitudinales de materiales translúcidos. Con ello se ha conseguido una iluminación natural, que permite ver con claridad suficiente en cualquier parte de la gran nave del taller cuya línea central de soportes la divide en dos crujías.

La luz cenital de los lucernarios facilita el poder realizar las operaciones de montaje en los sitios previstos para ello. Para mejorar la iluminación natural y artificial, el solado está constituido por una losa de base, de hormigón armado, a la que se le ha aplicado una capa de caucho de cloro pintada convenientemente para lograr una superficie de fácil reflexión que mejora las condiciones de iluminación.

La nave y servicios del taller se han dispuesto de tal forma que las reparaciones de automóviles marca Volkswagen se puedan La nave y servicios del taller se han dispuesto de tal forma
efectuar siguiendo los procedimientos de cadena de montaje.

Los coches entran canalizados por un lateral, siguen el curso interior del trazado de la cadena de montaje y salen reparados por el otro lado, que es el lateral de la nave.

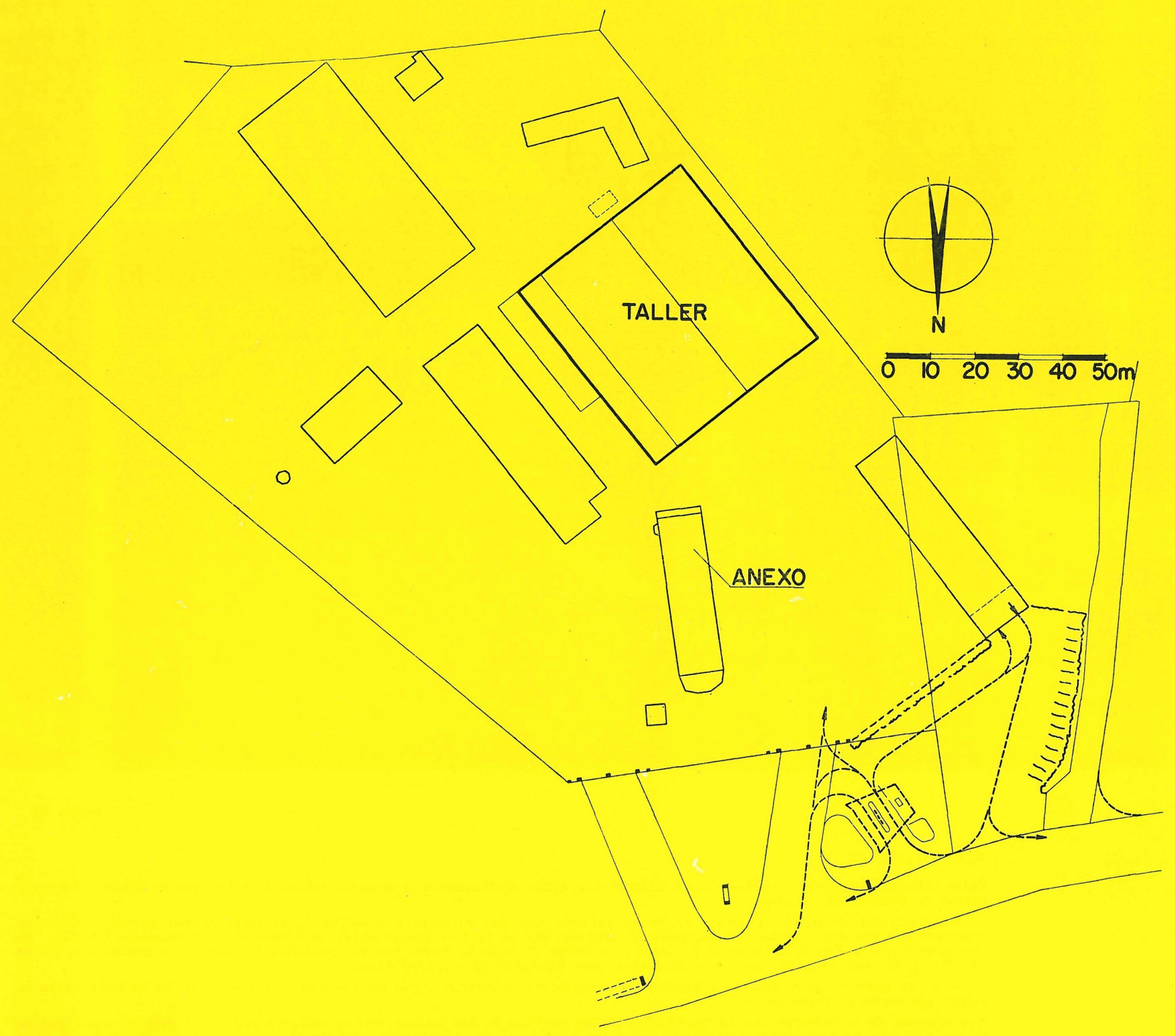

\section{planta general}



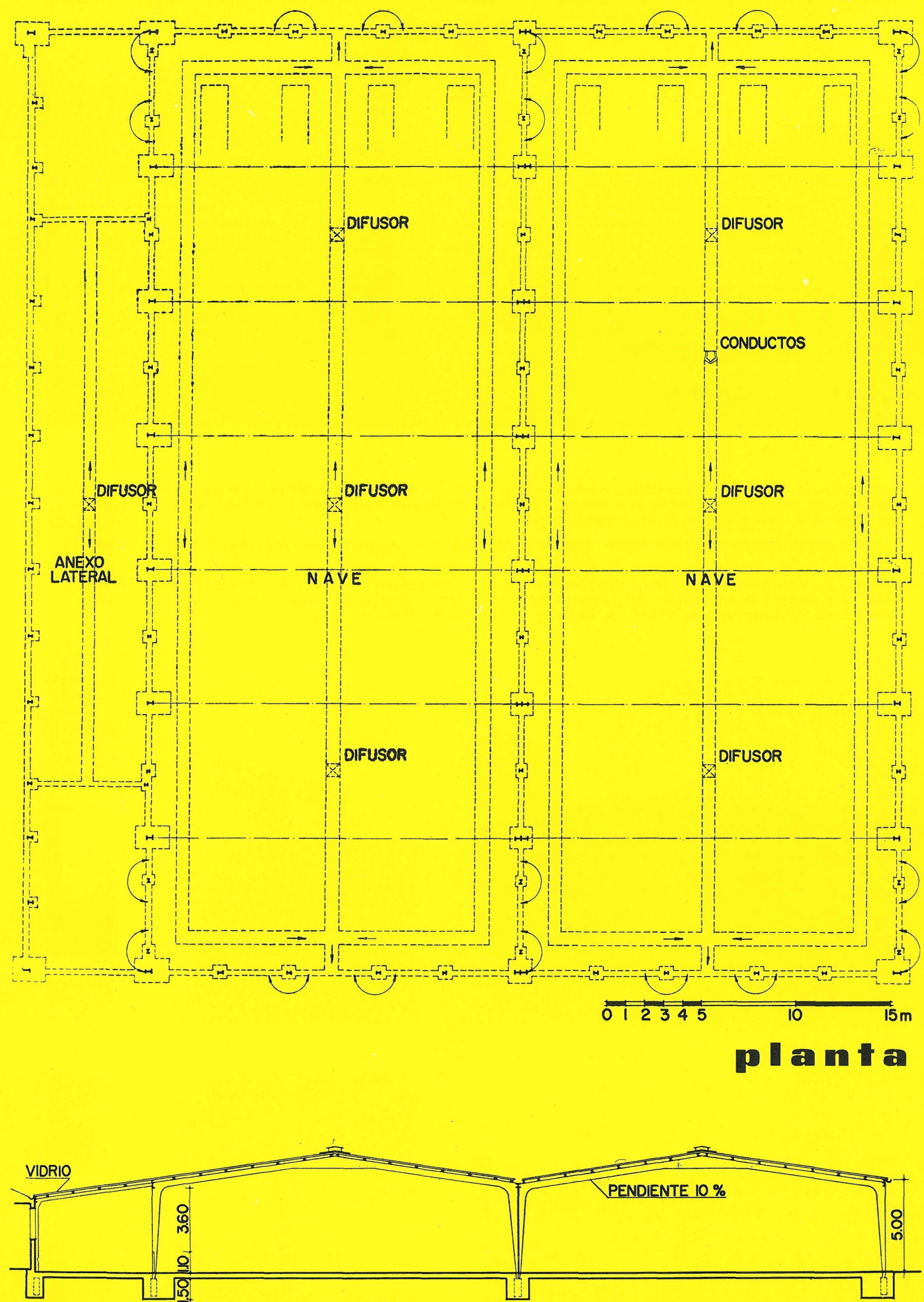

SECEÍ⿴囗十 


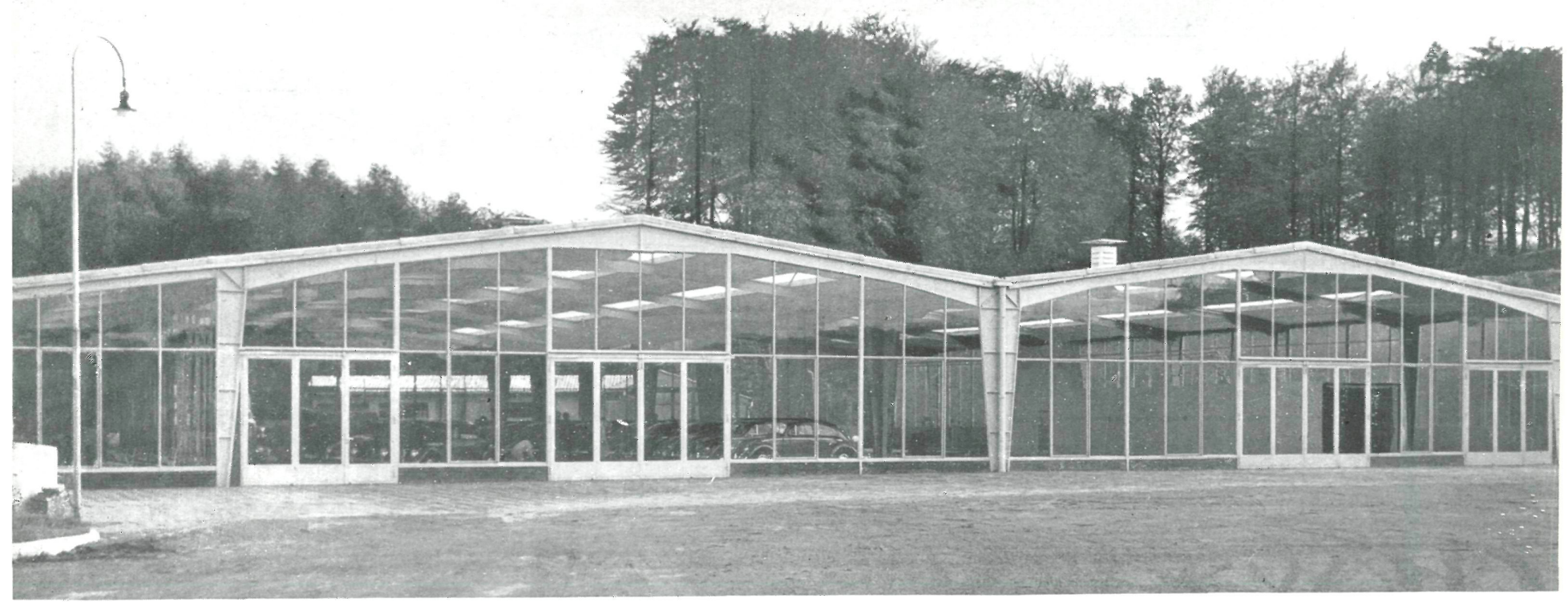

Considerando la estructura metálica como armazón resistente del edificio industrial o taller, su despiece se reduce a una serie de pórticos de dos tramos. Uno de estos tramos cubre toda la anchura de la nave prin. cipal, y el otro, más reducido, la de la nave lateral.

Los soportes son de chapa, de forma trapezoidal invertida y se apoyan sobre cimientos en forma de dado.

Los accesos, la disposición de servicios, iluminación y esbeltez de la estructura constituyen un conjunto moderno de arcuitectura industrial Complementa la serie de mejoras logradas una instalación de aire ca. liente, que asegura la calefacción del taller durante el invierno. Este aire caliente pasa, debidamente cana. lizaco, a los difusores que se hallan en la parte inferior de la cubierta.
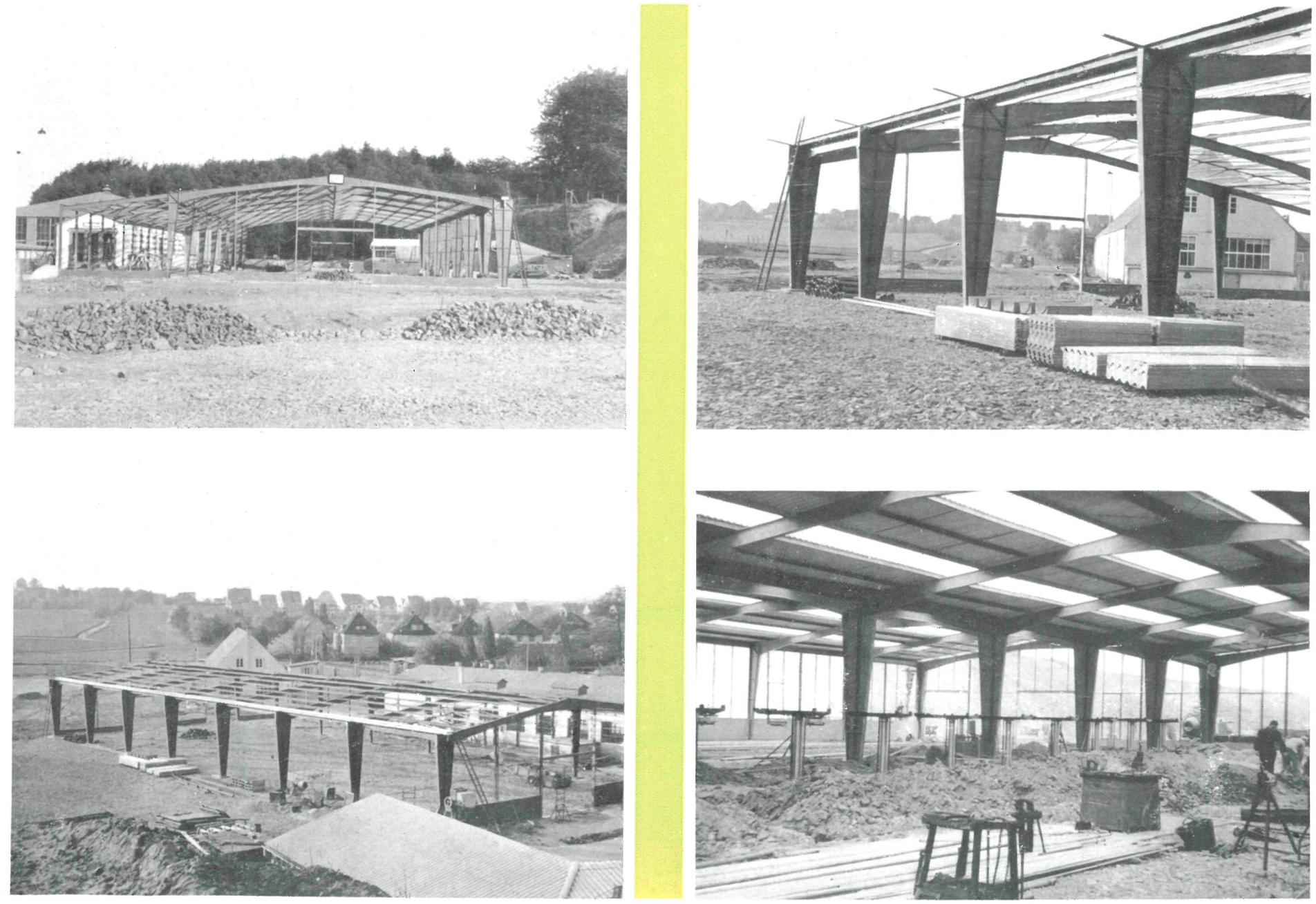
Vista parcial
de la gran nave.

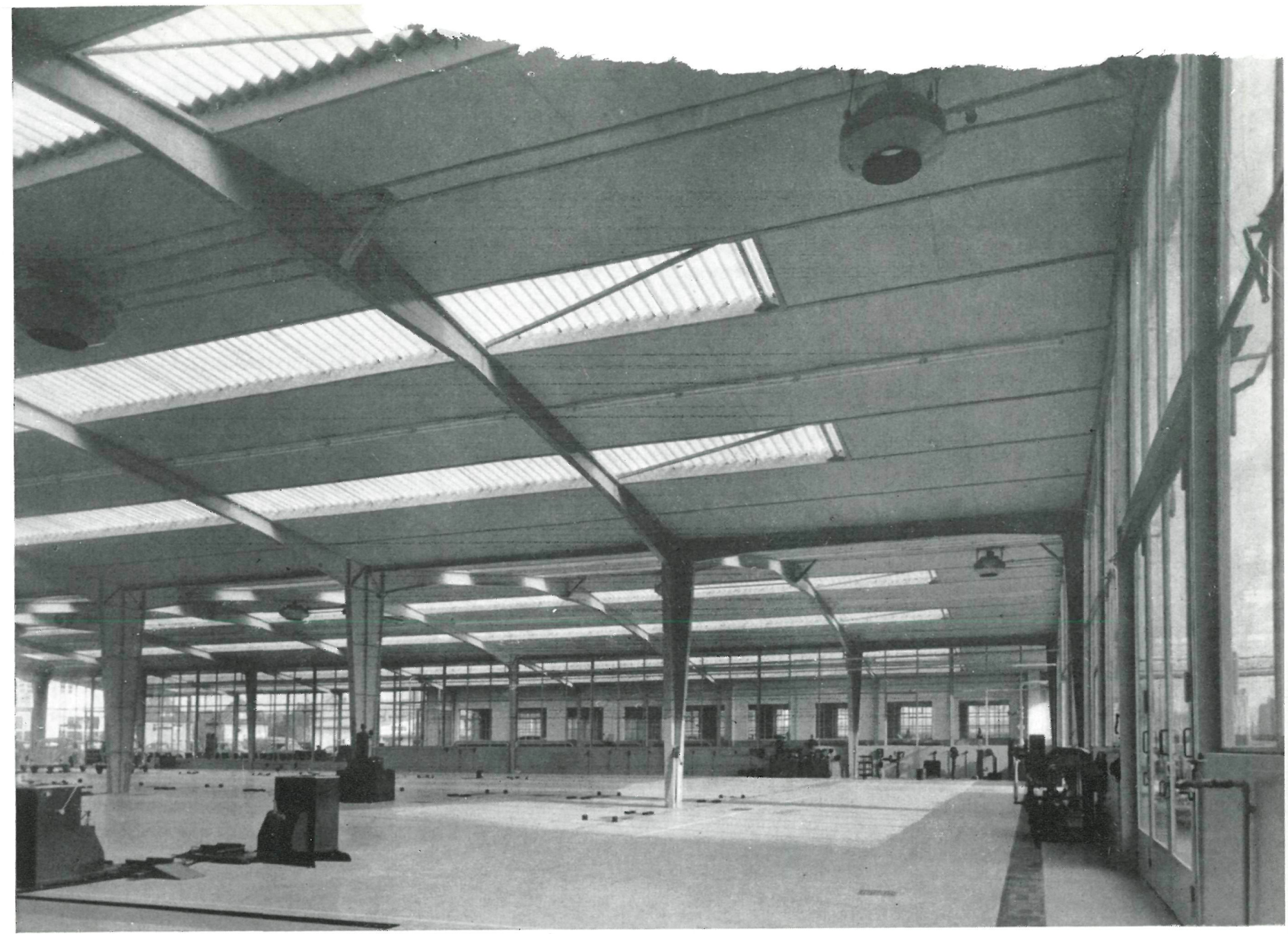

Fosas para

reparaciones

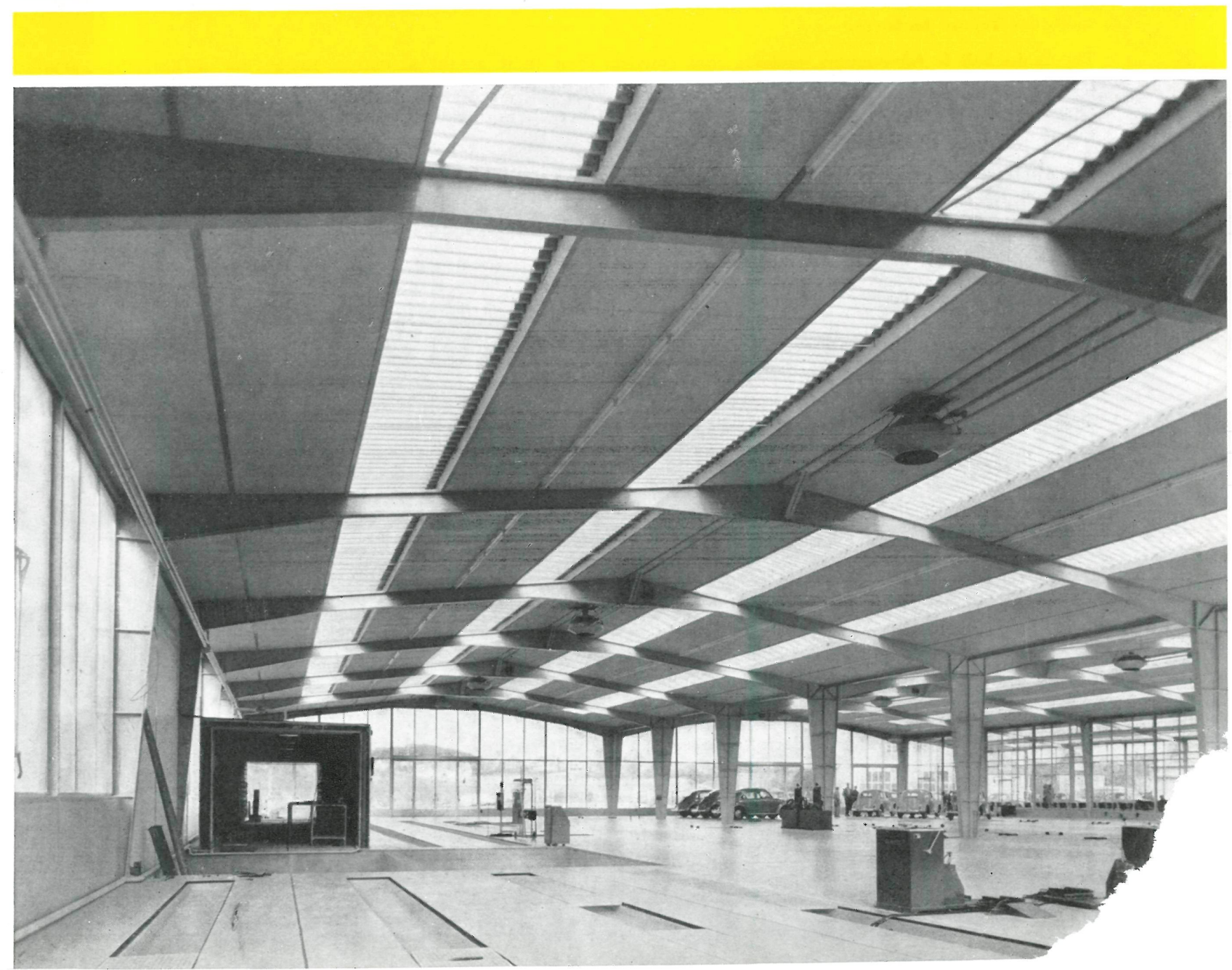




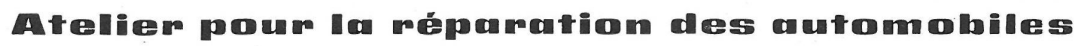

Adolf Ide, architecte

Ces ateliers, construits à Osnabrück (Allemagne), sont destinés à l'entreprise de réparation d'automobiles de tourisme Starke \& Sohn.

Le hall général, rectangulaire, a été couvert d'une structure métallique formée par de gracieux portiques sur lesquels sont placées les pannes et la couverture traditionnelle en plaques ondulées de fibrociment. De grandes verrières pour l'éclairage zénithal courent tout au long du hall, les murs latéraux et frontaux sont formés par des panneaux vitrés, ce qui, comme de juste, donne un éclairage extraordinaire.

On a prévu tout pour que les véhicules à réparer suivent un circuit continu depuis leur entrée jusqu'à leur sortie une fois réparés.

Les supports de la couverture sont les montants des portiques de deux tronçons qui constituent toute la structure résistante du hall. Les problèmes de ventilation, d'éclairage artificiel et de conditionnement intérieur ont été parfaitement étudiés.

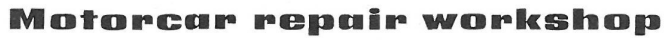

Adolf Ide, architect.

These workshops are in Osnabrück (Germany), and belong to the firm of Starke \& Sohn, which is in the motorcar repair business.

The main hall is rectangular, and its roof consists of a light metal structure made up of graceful portal frames, which support the longerons and roofing material; the latter being corrugated fibrocement sheeting. Large skylights run the length of the hall. The side and front walls are mainly glass sheets, thereby giving the workshop exceptional luminosity.

The roof is supported on the side members of the portal frames, which have two spans. Ventilation, artificial lighting and air conditioning are fully catered for.

Everything is so planned that the vehicles to be repaired shall follow a continuous circuit from the moment of entry until they go out fully repaired.

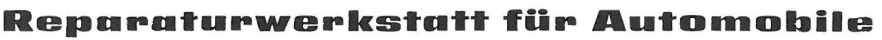

Adolf Ide, Architekt

Diese in Osnabrück (Deutschland) erbaute Werkstatt ist für das Unternehmen Starke \& Sohn bestimmt, das sich der Reparatur von leichten Automobilen widmet.

Die allgemeine Halle mit rechteckigem Grundriss wurde mit einem leichten Metall-Tragwerk bedeckt, das anmutige Rahmen bildet, über welche die Gurten und das Dachmaterial führen, das aus dem altherkömmlichen Wellenblech aus Asbestzement besteht. Grosse Leuchtkörper für die Deckenbeleuchtung sind längs der ganzen Halle angebracht. Die Umschliessung an den Seiten und an der Vorderfront erfolgt durch Glaswände, die natürlich eine ausserordentliche Helligkeit verschaffen.

Alles wurde derart vorbereitet, dass die zu reparierenden Fahrzeuge eine stetige Rundfahrt von der Ein-bis zur Ausfahrt des bereits reparierten Wagens zu machen haben.

Die Dachstützen bilden die Pfosten für die Rahmen von zwei Abschnitten, welche das ganze widerständige Tragwerk der Halle ausmachen. Alle Probleme der Belüftung, der künstlichen Beleuchtung und der inneren Beschaffenheit wurden vollkommen durchdacht. 